\title{
Estudo da estabilidade de polpas de frutas tropicais mistas congeladas utilizadas na formulação de bebidas
}

\author{
Study of the stability of frozen mixed tropical fruit pulps used in the formulation of \\ beverages
}

\author{
Ana Vânia Carvalho ${ }^{*}$, Rafaella de Andrade Mattietto', Jacqueline Chaves Beckman² \\ 1 Embrapa Amazônia Oriental, Laboratório de Agroindústria, Belém/PA - Brasil \\ 2 Universidade Federal do Pará (UFPa), Belém/PA - Brasil
}

\section{*Corresponding Author}

Ana Vânia Carvalho, Embrapa Amazônia Oriental, Laboratório de Agroindústria, Travessa Dr. Enéas Pinheiro, s/n, CEP: 66095-100, Belém/PA - Brasil, e-mail: ana-vania.carvalho@embrapa.br

Cite as: Study of the stability of frozen mixed tropical fruit pulps used in the formulation of beverages. Braz. J. Food Technol., v. 20, e2016023, 2017.

Received: Feb. 24, 2016; Accepted: Apr. 04, 2017

\section{Resumo}

O objetivo deste trabalho foi avaliar a estabilidade de duas polpas de frutas tropicais mistas, armazenadas sob congelamento $\left(-18{ }^{\circ} \mathrm{C}\right)$, durante 365 dias, a partir de sucos tropicais mistos: $\mathrm{F} 1$ - suco tropical misto de acerola $(10 \%)$, abacaxi (20\%), açaí (5\%), caju (5\%), cajá (5\%), camu-camu (5\%), água (43,1\%) e açúcar (6,9\%) e F2 - suco tropical misto de acerola (10\%), abacaxi (20\%), açaí (10\%), cajá (10\%), água (43,4\%) e açúcar (6,6\%). Análises físicas, químicas e de capacidade antioxidante foram realizadas a cada 45 dias. Os sucos tropicais mistos mostraram-se adequados em termos da legislação vigente no que se refere à acidez e aos sólidos solúveis. Quanto à presença de constituintes bioativos, o suco tropical misto com maior concentração de açaí e cajá apresentou os maiores teores de antocianinas totais e carotenoides totais. Depois de 365 dias de armazenamento congelado, as perdas observadas para o teor de carotenoides foram 2,80\% para a formulação F1 e 10,07\% para a formulação F2. Já para antocianinas totais, as perdas foram maiores, 44,35\% e 73,48\%, para as formulações F1 e F2, respectivamente. De maneira geral, observou-se que o armazenamento sob congelamento foi eficaz na manutenção das características químicas e de atividade antioxidante das polpas de frutas mistas estudadas, resultando, após o descongelamento e adição de água e sacarose, em sucos tropicais mistos com boa qualidade nutricional e funcional.

Palavras-chave: Caracterização físico-química; Constituintes bioativos; Sucos mistos; Armazenamento congelado.

\section{Abstract}

The aim of this study was evaluate two formulations of mixed tropical fruit pulps stored frozen $\left(-18^{\circ} \mathrm{C}\right)$ for 365 days as from the mixed tropical juices obtained from them: F1 - mixed tropical juice of acerola (10\%), pineapple (20\%), acai (5\%), cashew $(5 \%)$, yellow mombin (5\%), camu-camu (5\%), water (43.1\%) and sucrose (6.9\%); and F2 - mixed tropical juice of acerola (10\%), pineapple (20\%), acai (10\%), yellow mombin (10\%), water (43.4\%) and sucrose (6.6\%). Physical and chemical analyses and the antioxidant capacity were determined every 45 days. The tropical juices obtained were suitable in terms of current legislation referring to acidity and soluble solids. With respect to the presence of bioactive constituents, the mixed tropical juice with the highest concentrations of acai and yellow mombin showed the highest levels of total anthocyanins and total carotenoids. After 365 days of frozen storage, the losses of carotenoid contents were $2.80 \%$ for formulation $\mathrm{F} 1$ and $10.07 \%$ for formulation F2. As for the total anthocyanins, the losses were higher, $44.35 \%$ and $73.48 \%$ for formulations F1 and F2, respectively. In general, it was observed that frozen storage was effective in maintaining the chemical characteristics and antioxidant activity of the mixed fruit pulps studied, resulting, after defrosting and the addition of water and sucrose, in mixed tropical juices with good nutritional and functional quality.

Keywords: Physicochemical characterization; Bioactive constituents; Mixed juices; Frozen storage. 


\section{Introdução}

A busca por alimentos mais saudáveis tem movimentado um mercado crescente para frutas e sucos derivados de frutas, no qual se destacam as frutas nativas brasileiras por suas características diferenciadas de sabor e aroma, e por possuírem um nível elevado de constituintes bioativos (NAKANO et al., 2012). O desenvolvimento de sucos e polpas de frutas mistas tem atraído consumidores devido a essas características diferenciadas, melhorando a aceitação sensorial dos produtos, e incrementando sua composição quanto aos compostos de interesse para a saúde, em especial a capacidade antioxidante.

Barbosa (2010) menciona que a mistura de mais de uma fruta para produção de sucos e polpas é uma tendência observada no mercado. Esses produtos mistos apresentam uma série de vantagens, tais como melhoria das características nutricionais do produto final pela complementação de nutrientes fornecidos por diferentes frutas, bem como desenvolvimento de novos sabores, visando atender às expectativas dos consumidores.

De acordo com a legislação brasileira (MAPA Instrução Normativa n.01, de 2000), polpas de frutas são produtos não fermentados, não concentrados, não diluídos, obtidos de frutos polposos, por processo tecnológico adequado, com um teor mínimo de sólidos totais, provenientes da parte comestível do fruto. Quando originadas de duas ou mais frutas são classificadas como mistas (BRASIL, 2000).

Polpas de frutas mistas são encontradas no mercado brasileiro há alguns anos, tendo a praticidade como principal vantagem para estabelecimentos comerciais como restaurantes, padarias, lanchonetes, entre outros. Indústrias de processamento também contam com a vantagem de ter um produto misto congelado, utilizado como ingrediente para obtenção de outros produtos, sem ter que realizar etapas de processamento adicionais em função do número de frutas envolvidas.

Dentre as alternativas de conservação para uma polpa de fruta, o congelamento ainda é o procedimento mais utilizado. Segundo Maia et al. (2007), quando os alimentos congelados são processados, armazenados e manipulados de forma adequada, apresentam características sensoriais e nutricionais muito similares às que possuem antes do seu congelamento. O processo, portanto, é considerado um dos mais indicados para a preservação das propriedades químicas, nutricionais e sensoriais dos alimentos. Apesar disso, deve-se considerar que é quase impossível evitar mudanças na qualidade dos alimentos durante a aplicação de processos de conservação (ORDOÑEZ PEREDA, 2005).

Em polpas de frutas mistas, a composição química de cada fruta utilizada se soma e afeta as características finais dos produtos obtidos, principalmente se a escolha das frutas é voltada para um maior potencial antioxidante em bebidas. Nesse contexto, é fundamental a avaliação da qualidade das polpas mistas durante o período de vida de prateleira (geralmente um ano sob congelamento). A avaliação das características físico-químicas, presença de constituintes bioativos e respectivas perdas ao longo da estocagem congelada das polpas está relacionada à estabilidade de bebidas obtidas a partir delas.

Assim, o objetivo deste trabalho foi avaliar a estabilidade física, química e de capacidade antioxidante, de duas polpas mistas de frutas tropicais (acerola, abacaxi, açaí, caju, cajá e camu-camu) no armazenamento congelado por 365 dias, a partir de formulações de bebidas mistas.

\section{Material e métodos}

Anteriormente à execução do presente estudo, a pesquisa realizada por Pereira et al. (2014) forneceu as condições otimizadas para a preparação das polpas mistas. Foram preparadas, portanto, as seguintes polpas mistas, codificadas como: P1 - polpa tropical mista composta por acerola (10\%), abacaxi (20\%), açaí (5\%), caju (5\%), cajá $(5 \%)$ e camu-camu (5\%); P2 - polpa tropical mista composta por acerola (10\%), abacaxi (20\%), açaí (10\%) e cajá (10\%). A escolha das frutas para compor as duas formulações, selecionadas do estudo de Pereira et al. (2014), teve por objetivo obter polpas de frutas mistas ricas em constituintes bioativos, para serem utilizadas no preparo de bebidas antioxidantes.

Para a preparação das polpas mistas (P1 e P2) foram empregadas polpas não pasteurizadas e congeladas, de acerola, abacaxi, caju e cajá adquiridas no mercado local de Fortaleza-CE e polpas de camu-camu e açaí procedentes do comércio local de Belém-PA.

A etapa de mistura para obtenção das polpas de frutas mistas foi feita em um moinho coloidal vibratório (METEOR, REX, Brasil) durante 4 minutos. Em seguida, foi realizado o envase em embalagens de polietileno de $1 \mathrm{~kg}$ (240 mm x $340 \mathrm{~mm} \times 0,15 \mathrm{~mm}$ ), sendo imediatamente seladas em seladora manual (SULPAK, SP 700 T, Brasil) e armazenadas a $-18^{\circ} \mathrm{C} \pm 3^{\circ} \mathrm{C}$, em câmara frigorífica. Como as polpas de frutas mistas foram desenvolvidas com o objetivo de gerar bebidas semiprontas para o consumo, isto é, com o consumidor adicionando-lhes somente água e sacarose, a caracterização física e físico-química e a capacidade antioxidante foram realizadas após o descongelamento e formulação das polpas mistas. A adição de água e açúcar às polpas na elaboração das bebidas mistas foi realizada de forma a resultar em teor de sólidos solúveis final na faixa de $12^{\circ}$ Brix, de acordo com as formulações propostas por Pereira et al. (2014).

Assim, as polpas de frutas P1 e P2, após o descongelamento e adição de água e açúcar, passaram a gerar as formulações de suco tropical misto F1: suco tropical misto de acerola (10\%), abacaxi (20\%), açaí (5\%), 
caju (5\%), cajá (5\%), camu-camu (5\%), água $(43,1 \%)$ e açúcar (6,9\%) e F2: suco tropical misto de acerola (10\%), abacaxi (20\%), açaí (10\%), cajá (10\%), água (43,4\%) e açúcar (6,6\%). As formulações (F1 e F2) de sucos mistos foram analisadas a cada 45 dias, pelo período de um ano, sendo que foram preparadas nos dias de análise.

As duas formulações mistas (F1 e F2) foram caracterizadas quanto a: $\mathrm{pH}$ (método n 981.12 da AOAC, CUNNIFF, 1997); acidez titulável (método n 942.15B da AOAC, CUNNIFF, 1997); sólidos solúveis (método n 932.12 da AOAC, CUNNIFF, 1997); cor instrumental (determinada em espectrofotômetro HUNTERLAB/COLORQUEST-XE (EUA) com leituras realizadas em sistema $\operatorname{CIELAB}\left(\mathrm{L}^{*}, \mathrm{a}^{*} \mathrm{e} \mathrm{b}^{*}\right)$ ); açúcares totais (por LANE e EYNON, 1934 (titulação de oxirredução)), segundo AOAC (WILLIANS, 1984). Para a análise de cor foi realizado o cálculo da diferença total de $\operatorname{cor}(\Delta \mathrm{E})$ entre a amostra no tempo zero e nos demais tempos de acompanhamento, utilizando-se a Fórmula 1:

$$
\Delta \mathrm{E}=\left[\Delta \mathrm{L}^{* 2}+\Delta \mathrm{a}{ }^{* 2}+\Delta \mathrm{b}^{* 2}\right]^{1 / 2}
$$

Onde os $\Delta \mathrm{L}^{*}, \Delta \mathrm{a}^{*}$ e $\Delta \mathrm{b}^{*}$ representaram a diferença entre o valor obtido no período de armazenamento em questão e os valores iniciais, para cada parâmetro.

O teor de carotenoides totais (extração e quantificação dos carotenoides), nas duas formulações mistas (F1 e F2), foi determinado de acordo com metodologia de RodriguezAmaya (1999). Para o cálculo de carotenoides totais empregaram-se os valores de absorbância no comprimento de onda (450 nm) e coeficiente de absorção (2592) para o $\beta$-caroteno, expressando-se o resultado de carotenoides totais em $\mu \mathrm{g} \mathrm{g}^{1}$.

O teor de antocianinas totais, nas formulações F1 e F2, foi determinado pelo método de $\mathrm{pH}$ diferencial, conforme descrito por Giusti e Wrolstad (2001). Os extratos aquosos foram apropriadamente diluídos em dois tampões: cloreto de potássio 0,025 M, pH 1 e acetato de sódio 0,4 M, pH 4,5. Foram feitas medidas de absorbância a $510 \mathrm{~nm}$ e $700 \mathrm{~nm}$ em espectrofotômetro (SHIMADZU, UV, 160-A, Japão), em cubetas de $1 \mathrm{~cm}$ de largura. $O$ teor dos pigmentos foi calculado considerando a absortividade molar (e) de $26900 \mathrm{~L} \mathrm{~mol}^{-1} \mathrm{~cm}^{-1}$, peso molecular de 449,2 g/mol da cianidina 3-glicosídeo e os resultados foram expressos como mg de cianidina 3-glicosídeo por 100 mL de polpa.

Para a determinação da atividade antioxidante total em F1 e F2, inicialmente procedeu-se à extração de antioxidantes, seguindo o procedimento desenvolvido por Larrauri et al. (1997) com algumas modificações descritas a seguir: as bebidas mistas (F1 e F2) foram pesadas (g) em tubos de centrífuga e extraídas sequencialmente com $40 \mathrm{~mL}$ de metanol/água (50:50, v/v) à temperatura ambiente por $1 \mathrm{~h}$. Os tubos foram centrifugados (EPPENDORF, 5804, Alemanha) a $20.000 \mathrm{~g}$ for 20 min e o sobrenadante foi recolhido. Adicionaram-se $40 \mathrm{ml}$ de acetona/água
(70:30, v/v) no resíduo em temperatura ambiente por $1 \mathrm{~h}$ e, a seguir, centrifugou-se a amostra nas mesmas condições citadas anteriormente. Os extratos de metanol e acetona foram combinados e ajustados para $100 \mathrm{~mL}$ com água destilada e usados para determinar a atividade antioxidante, de acordo com os métodos citados abaixo:

Determinação da capacidade antioxidante total pelo método ABTS: baseada no método desenvolvido por Miller et al. (1993), com modificações de acordo com Rufino et al. (2007). O radical ABTS ${ }^{\bullet+}$ foi produzido pela reação de 7 mM de solução estoque de ABTS com 145 mM de persulfato de potássio no escuro e em temperatura ambiente por 12-16 h antes do uso. A solução ABTS foi diluída com etanol para uma absorbância de $0.70 \pm 0.02$ a $734 \mathrm{~nm}$. Depois de pipetar $30 \mu \mathrm{L}$ de amostra ou o padrão trolox, adicionaram-se $3 \mathrm{ml}$ de solução diluída de ABTS•+ e as absorbâncias foram lidas após 6 min da mistura em espectrofotômetro (SHIMADZU UV, 160-A, Japão). Soluções etanólicas com concentrações conhecidas de trolox foram usadas para a calibração e os resultados foram expressos em $\mu$ mol trolox $g^{-1}$ amostra.

Determinação da capacidade antioxidante total pelo método DPPH•: baseada no método de Brand-Wiliams et al. (1995). Uma solução de metanol contendo 0,06 mM DPPH• foi preparada e uma alíquota de $100 \mu \mathrm{L}$ de extrato foi adicionado a 3,9 ml da solução de metanol. A diminuição da absorbância a $515 \mathrm{~nm}$ foi determinada em intervalos de 1 minuto durante os primeiros 10 minutos e, então, a cada 5 minutos, até a estabilização. A capacidade antioxidante foi expressa como a concentração de antioxidante requerida para reduzir a quantidade original de radical livre para 50\% (EC50) e os resultados foram expressos como g amostra $\mathrm{g}^{-1} \mathrm{DPPH} \bullet$

Os resultados das características avaliadas para as bebidas mistas (F1 e F2) durante 365 dias de armazenamento foram submetidos à análise de variância e as médias, quando significativas, comparadas pelo Teste de Tukey a 5\% de significância, com auxílio do programa SAS 8.0 (SAS INSTITUTE, 1999).

\section{Resultados e discussão}

Na Tabela 1, são apresentados os resultados das análises de $\mathrm{pH}$, acidez titulável, sólidos solúveis, açúcares totais e cor instrumental para as duas formulações de suco tropical misto, preparadas com polpas de frutas mistas armazenadas sob congelamento, ao longo de 365 dias.

Observando-se o tempo zero, nota-se que as duas formulações podem ser consideradas ácidas, pelos valores de $\mathrm{pH}$ e acidez encontrados, sendo observada diferença significativa somente para a análise de acidez titulável. Apesar da diferença observada, os valores são muito próximos, o que nos permite concluir que a presença de caju e camu-camu na formulação F1 não tornou a bebida muito mais ácida, mesmo sendo a polpa 


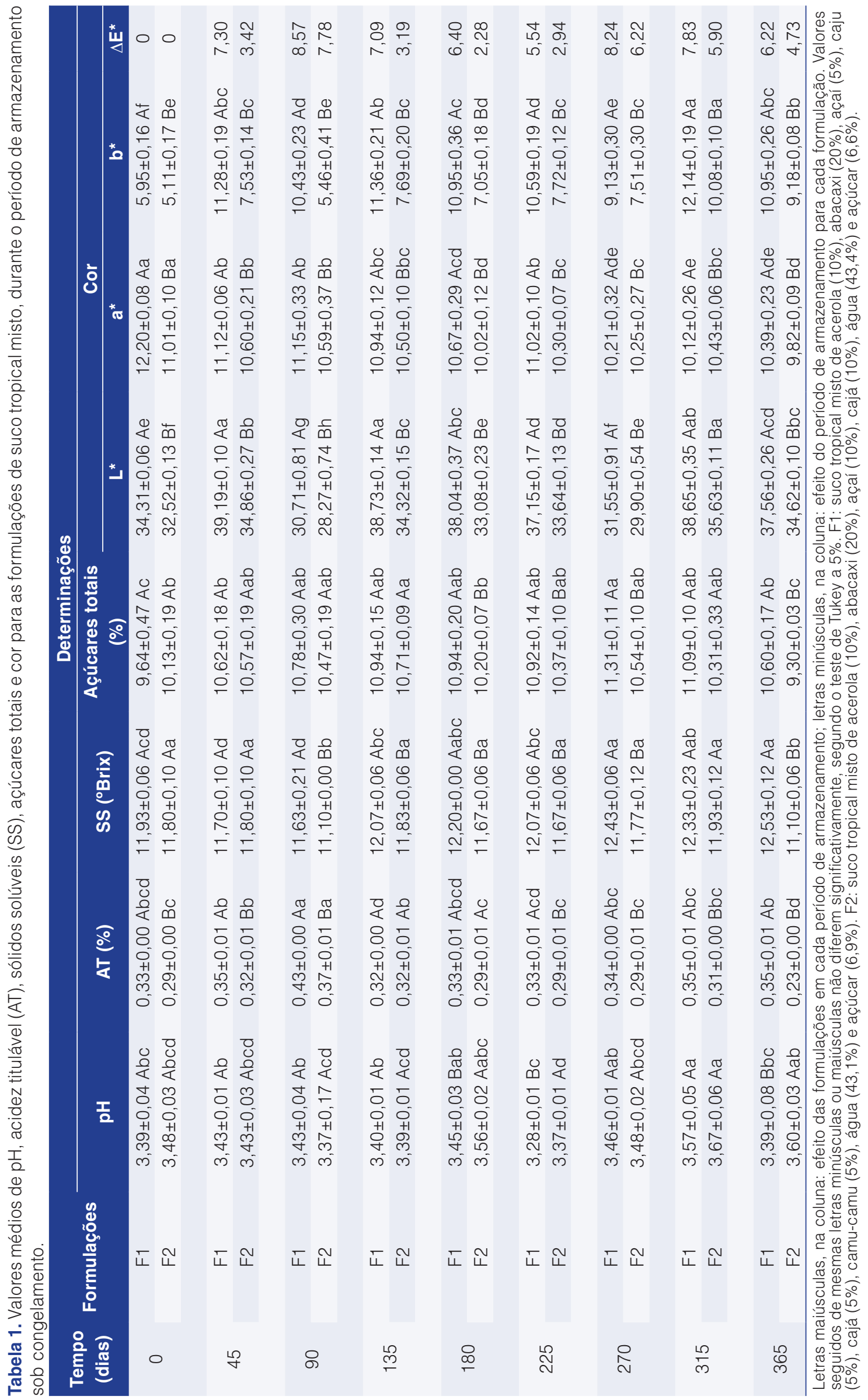


do camu-camu ácida, com valores variando de 1,8 a 3,8\% (HERNÁNDEZ et al., 2011).

Em relação ao período de armazenamento, observaram-se oscilações nas características físicas e físico-químicas avaliadas, porém sem uma tendência definida. Os valores de sólidos solúveis se mostraram estáveis, para as duas formulações avaliadas, mesmo após um ano de armazenamento sob congelamento. Igualmente não houve variações significativas nos valores de $\mathrm{pH}$ e acidez titulável da formulação F1. Já em relação à formulação F2, apenas a acidez titulável apresentou decréscimo significativo; contudo a variação pode ser considerada irrelevante quando se considera o tempo de armazenamento (365 dias), indicando que o congelamento, como esperado, é capaz de manter a qualidade físico-química de polpas de frutas.

Os parâmetros acidez titulável e sólidos solúveis totais, em todos os períodos estudados, permaneceram de acordo com os padrões de identidade e qualidade para sucos tropicais, que estabelecem valores mínimos de $0,20 \%$ de acidez titulável em ácido cítrico e $11^{\circ}$ Brix para sólidos solúveis (BRASIL, 2003).

Em estudo realizado por Bezerra et al. (2013) sobre o comportamento reológico do suco tropical misto elaborado pela combinação de polpas de acerola, maracujá e taperebá, os autores observaram valores de sólidos solúveis de $18,2^{\circ}$ Brix, acidez titulável de 0,72\% e pH de 3,3. Já Neves et al. (2011) verificaram, para néctares e blends preparados a partir de frutos tropicais e amazônicos, valores variando de 7,70 Brix a 13,5 Brix para sólidos solúveis, 0,56 a 4,16\% de acidez em ácido cítrico e pH de 2,9 a 4,4, cujas variações observadas são devidas às diferentes combinações de frutas utilizadas, uma vez que cada uma possui suas características particulares. Observou-se que esses valores relatados na literatura estão próximos aos dados obtidos no presente estudo (Tabela 1).

Para o teor de açúcares totais, os valores variaram de 9,30 a 11,31\% para as formulações ao longo do tempo, sem, portanto, observar-se uma tendência definida. Esses valores estão de acordo com os padrões de identidade e qualidade para alguns sucos tropicais adoçados, que estabelecem valores mínimos de $8 \%$ de açúcares totais para sucos tropicais de abacaxi, cajá e caju, e valor mínimo de $7 \%$ de açúcares totais para suco tropical de acerola (BRASIL, 2003).

Para as duas formulações de suco tropical misto, observaram-se valores do parâmetro $L^{*}$ inferiores a 50 e valores positivos para as coordenadas cromáticas $a^{*} e$ $b^{\star}$, o que as caracteriza como bebidas mais escuras e de coloração amarelo-avermelhada. Quando se comparou as duas formulações estudadas, observou-se que F1 apresentou-se, durante todo o período de armazenamento, mais clara que F2, o que era de certa forma esperado, uma vez que F2 possui maior quantidade de polpa de açaí, e, portanto, maior quantidade de pigmentos escuros (antocianinas). Além disso, F1 apresentou valores superiores para as coordenadas $a^{*} e b^{*}$, indicando que a mesma apresentava coloração amarelo-avermelhada ligeiramente mais intensa que a F2, a qual não possuía, em sua formulação, presença de polpas de caju e camu-camu. As polpas de caju e camu-camu apresentam colorações amarelada e rósea, respectivamente, o que pode ter contribuído para a ligeira diferença de coloração entre os dois sucos tropicais estudados.

Ainda com relação à análise de cor, os valores de $\Delta \mathrm{E}^{\star}$ mostraram variação total de cor entre as amostras em relação a um padrão, no caso, o tempo zero. Para as bebidas mistas, elaboradas a partir das polpas mistas armazenadas sob congelamento, verificou-se, já a partir de 45 dias de armazenamento, alteração na coloração dos produtos, com valores médios de $\Delta \mathrm{E}^{*}$ de 7,30 e 3,42 para as formulações F1 e F2, respectivamente.

$\mathrm{O} \Delta \mathrm{E}^{*}$ para a $\mathrm{F} 1$ foi maior em todos os tempos avaliados, mesmo esta formulação apresentando menores concentrações de carotenoides e antocianinas (Tabela 2), que são pigmentos instáveis na estocagem. Teoricamente, portanto, a formulação F2 poderia apresentar maior variação de cor. Entretanto, em 45 dias, ocorreu uma perda significativa de antocianinas em F1 (41,16\%). Este fato, relacionado com o ligeiro aumento de $\mathrm{pH}$ observado para essa formulação (Tabela 1), pode explicar a maior variação de cor encontrada. Segundo Lopes et al. (2007), a sensibilidade ao $\mathrm{pH}$ é o principal fator limitante no processamento e utilização das antocianinas, afetando a cor e a estabilidade química.

$\mathrm{Na}$ Tabela 2, são apresentados os resultados da caracterização química e da capacidade antioxidante das formulações de suco tropical misto. Observaram-se variações significativas nas características avaliadas, tanto entre as duas formulações estudadas quanto ao longo do período de armazenamento.

Os sucos tropicais mistos de acerola, abacaxi, açaí, caju, cajá e camu-camu (F1) e acerola, abacaxi, açaí e cajá (F2) podem ser considerados como produtos novos, não tendo referência de comparação de suas caracterizações químicas na literatura. Entretanto, os valores apresentados na Tabela 2 estão dentro da faixa esperada para produtos à base de frutas (FREITAS et al., 2006; MATTIETTO et al., 2007; CARVALHO et al., 2011; VEGARA et al., 2013).

Observou-se que a maior concentração de cajá na formulação F2 resultou em maiores teores de carotenoides totais ao longo de todo o período de armazenamento. Para as duas formulações, após 365 dias de armazenamento sob congelamento, as perdas observadas nos teores de carotenoides foram 2,80\% para F1 e 10,07\% para F2. Os valores encontrados para as duas formulações, ao 
Estudo da estabilidade de polpas de frutas tropicais mistas congeladas utilizadas na formulação de bebidas Carvalho, A. V. et al.

Tabela 2. Constituintes bioativos e capacidade antioxidante de duas formulações de suco tropical misto, durante o período de armazenamento sob congelamento.

\begin{tabular}{|c|c|c|c|c|c|}
\hline \multirow[b]{2}{*}{ Tempo (dias) } & \multicolumn{5}{|c|}{ Determinações } \\
\hline & Formulações & $\begin{array}{c}\text { Carotenoides totais } \\
\text { ( } \mu \mathrm{g} \mathrm{g}^{-1} \text { amostra) }\end{array}$ & $\begin{array}{l}\text { Antocianinas totais } \\
\text { (mg } 100 \mathrm{~mL}^{-1} \mathrm{de} \\
\text { amostra) }\end{array}$ & $\begin{array}{c}\text { ABTS }{ }^{++} \\
\left(\mu \mathrm{M} \text { trolox } \mathrm{g}^{-1}\right. \\
\text { amostra) }\end{array}$ & $\begin{array}{c}\text { DPPH } \\
\text { (g amostra } \text { g }^{-1} \text { DPPH) }\end{array}$ \\
\hline \multirow{2}{*}{0} & $\mathrm{~F} 1$ & $5,34 \pm 0,29 \mathrm{Bab}$ & $5,66 \pm 0,37 \mathrm{Ba}$ & $13,54 \pm 0,09 \mathrm{Aa}$ & $6159,31 \pm 214,81 \mathrm{Be}$ \\
\hline & $\mathrm{F} 2$ & $8,24 \pm 0,32 \mathrm{Aab}$ & $10,07 \pm 0,90 \mathrm{Aa}$ & $7,85 \pm 0,36 \mathrm{Bc}$ & $7934,27 \pm 168,64 \mathrm{Ae}$ \\
\hline \multirow{2}{*}{45} & $\mathrm{~F} 1$ & $4,99 \pm 0,37 \mathrm{Bab}$ & $3,33 \pm 0,15 \mathrm{Bbc}$ & $10,14 \pm 2,05 \mathrm{Abc}$ & $7305,92 \pm 314,86 \mathrm{Ac}$ \\
\hline & $\mathrm{F} 2$ & $9,11 \pm 0,86 \mathrm{Aa}$ & $6,98 \pm 0,08 \mathrm{Ab}$ & $8,59 \pm 0,46 \mathrm{Abc}$ & $8453,58 \pm 742,96$ Acde \\
\hline \multirow{2}{*}{90} & $\mathrm{~F} 1$ & $5,44 \pm 0,06 \mathrm{Ba}$ & $4,02 \pm 0,014 \mathrm{Bb}$ & $9,88 \pm 1,58 \mathrm{Abc}$ & $6738,97 \pm 128,61 \mathrm{Bd}$ \\
\hline & $\mathrm{F} 2$ & $7,89 \pm 0,31 \mathrm{Aab}$ & $7,83 \pm 0,57 \mathrm{Ab}$ & $10,19 \pm 0,43 \mathrm{Aa}$ & $9176,59 \pm 248,96 \mathrm{Abc}$ \\
\hline \multirow{2}{*}{135} & $\mathrm{~F} 1$ & $5,49 \pm 0,18 \mathrm{Ba}$ & $2,73 \pm 0,18 \mathrm{Bcd}$ & $7,76 \pm 0,95 \mathrm{Abc}$ & $8071,49 \pm 49,21 \mathrm{Bb}$ \\
\hline & $\mathrm{F} 2$ & $8,64 \pm 0,38 \mathrm{Aab}$ & $7,23 \pm 0,09 \mathrm{Ab}$ & $8,59 \pm 0,46 \mathrm{Abc}$ & $9846,87 \pm 336,22 \mathrm{Aab}$ \\
\hline \multirow{2}{*}{180} & F1 & $4,33 \pm 0,21 \mathrm{Bc}$ & $3,16 \pm 0,02 \mathrm{Bcd}$ & $7,28 \pm 0,19 \mathrm{Ac}$ & $8593,75 \pm 95,62 \mathrm{Ba}$ \\
\hline & $\mathrm{F} 2$ & $7,39 \pm 0,42 \mathrm{Ab}$ & $7,13 \pm 0,26 \mathrm{Ab}$ & $8,07 \pm 1,01 \mathrm{Abc}$ & $10148,14 \pm 262,12 \mathrm{Aa}$ \\
\hline \multirow{2}{*}{225} & F1 & $5,12 \pm 0,32 \mathrm{Bab}$ & $3,08 \pm 0,18 \mathrm{Bbc}$ & $9,84 \pm 0,36 \mathrm{Ab}$ & $7213,14 \pm 277,77 \mathrm{Bcd}$ \\
\hline & $\mathrm{F} 2$ & $7,62 \pm 0,53 \mathrm{Ab}$ & $7,12 \pm 0,63 \mathrm{Ab}$ & $9,96 \pm 0,62 \mathrm{Aab}$ & $8378,64 \pm 303,64$ Acde \\
\hline \multirow{2}{*}{270} & F1 & $4,84 \pm 0,14 \mathrm{Bbc}$ & $3,11 \pm 0,01 \mathrm{Bcd}$ & $8,30 \pm 0,31 \mathrm{Abc}$ & $6998,41 \pm 93,84 \mathrm{Bcd}$ \\
\hline & $\mathrm{F} 2$ & $7,53 \pm 0,23 \mathrm{Ab}$ & $6,85 \pm 0,08 \mathrm{Ab}$ & $7,34 \pm 0,31 \mathrm{Ac}$ & $8154,44 \pm 38,46$ Ade \\
\hline \multirow{2}{*}{315} & $\mathrm{~F} 1$ & $4,53 \pm 0,08 \mathrm{Bc}$ & $3,33 \pm 0,14 \mathrm{Bbc}$ & $8,74 \pm 0,25 \mathrm{Abc}$ & $8744,28 \pm 33,13 \mathrm{Ba}$ \\
\hline & $\mathrm{F} 2$ & $5,74 \pm 0,83 \mathrm{Ac}$ & $6,98 \pm 0,11 \mathrm{Ab}$ & $7,66 \pm 0,65 \mathrm{Ac}$ & $8874,14 \pm 19,41$ Acd \\
\hline \multirow{2}{*}{365} & $\mathrm{~F} 1$ & $5,19 \pm 0,04 \mathrm{Bab}$ & $2,51 \pm 0,14 \mathrm{Bd}$ & $9,03 \pm 0,40 \mathrm{Abc}$ & $8681,78 \pm 27,25 \mathrm{Aa}$ \\
\hline & $\mathrm{F} 2$ & $7,41 \pm 0,18 \mathrm{Ab}$ & $7,40 \pm 0,03 \mathrm{Ab}$ & $8,31 \pm 0,31 \mathrm{Abc}$ & $8736,33 \pm 44,98$ Acde \\
\hline
\end{tabular}

Onde: letras maiúsculas, na coluna: efeito das formulações em cada período de armazenamento; letras minúsculas, na coluna: efeito do período de armazenamento para cada formulação. Valores seguidos de mesmas letras minúsculas ou maiúsculas não diferem significativamente, segundo o teste de Tukey a 5\%. F1: suco tropical misto de acerola (10\%), abacaxi (20\%), açaí (5\%), caju (5\%), cajá (5\%), camu-camu (5\%), água (43,1\%) e açúcar (6,9\%). F2: suco tropical misto de acerola (10\%), abacaxi (20\%), açaí (10\%), cajá (10\%), água (43,4\%) e açúcar (6,6\%).

final do período de armazenamento, não apresentaram diferença significativa pelo teste estatístico aplicado. Hamano e Mercadante (2001), em estudo da composição de carotenoides em produtos comerciais de cajá, encontraram o teor de 16,71 $\mu \mathrm{g} \mathrm{g}^{-1}$ em suco integral de cajá. Mattietto et al. (2007), em estudo sobre a estabilidade do néctar misto de cajá e umbu, observaram valor de 9,85 $\mu \mathrm{g} \mathrm{g}^{-1}$ para carotenoides totais, valor próximo aos obtidos no presente trabalho.

A formulação F2 destacou-se em relação ao teor de antocianinas totais. A maior concentração de açaí na formulação resultou em um aumento de $77,91 \%$ no teor de antocianinas totais em relação à formulação F1. Observou-se, também, que, durante a estocagem, as antocianinas foram mais instáveis que os carotenoides, com perdas de $44,35 \%$ e $73,48 \%$ para F1 e F2, respectivamente. Em estudo sobre a estabilidade das antocianinas em suco tropical de acerola adoçado e envasado pelo processo asséptico, Freitas et al. (2006) constataram, ao final de 350 dias de armazenamento, uma redução de 86,89\% no teor de antocianinas. Os autores alegaram que essa acentuada perda pode ter sido favorecida pela variação do $\mathrm{pH}$ dos sucos durante o armazenamento, além de uma possível entrada de oxigênio atmosférico para o interior da embalagem e uma suposta regeneração de enzimas no suco.

Devido à diversidade das estruturas químicas contidas nos extratos vegetais, as variações dos constituintes e a sensibilidade às condições de extração, é importante avaliar a atividade antioxidante por mais de um método, visto que é difícil encontrar um único método que seja adequado para a extração de todos os grupos de constituintes com atividade antioxidante (REZAIRE et al., 2014; PAZ et al., 2009). Assim, a atividade antioxidante neste estudo foi determinada pelos métodos $\mathrm{ABTS}^{\bullet+}$ e DPPH $\bullet$. 
Em relação à capacidade antioxidante determinada pelo método $\mathrm{ABTS}^{+}$, observou-se diferença significativa entre as formulações F1 e F2 no tempo zero, no qual a F1 apresentou uma maior atividade antioxidante, de 13,54 $\mu \mathrm{M}$ trolox $\mathrm{g}^{-1}$. Após 365 dias, observou-se queda de $33,16 \%$ na capacidade antioxidante da formulação F1. Quanto à formulação F2, não se observou um comportamento claro, com aumentos e decréscimos dos valores, sem uma tendência definida, impossibilitando uma conclusão.

Os resultados expressos em EC50 indicaram a concentração de extrato capaz de reagir com 50\% do radical presente na solução de DPPH $\bullet$. Portanto, quanto menor o valor do EC50, maior será a atividade antioxidante do extrato analisado. Assim, observou-se que o suco tropical misto F1 apresentou uma capacidade antioxidante significativamente maior que a da formulação de suco misto F2, com exceção do tempo 45 e 365 dias. Ao longo do período de armazenamento, verificaram-se pequenas oscilações, contudo sem uma tendência definida. O mesmo comportamento foi observado para a capacidade antioxidante determinada pelo método ABTS •+.

De maneira geral, os valores de capacidade antioxidante obtidos no presente estudo variaram de $6.159,31$ a $10.148,14 \mathrm{~g} \mathrm{~g}^{-1}$ (método DPPH) e de 7,28 a $13,58 \mu \mathrm{M}$ trolox $\mathrm{g}^{-1}$ (método ABTS ${ }^{\bullet}$ ). Rufino et al. (2010), em estudo sobre a capacidade antioxidante de frutas tropicais brasileiras não tradicionais, verificaram valores de $4.264 \mathrm{~g} \mathrm{~g}^{-1} \mathrm{DPPH} \bullet$ e 15,1 $\mathrm{\mu M}$ trolox $\mathrm{g}^{-1}$ para açaí, $670 \mathrm{~g} \mathrm{~g}^{-1} \mathrm{DPPH} \bullet$ e 96,6 $\mu \mathrm{M}$ trolox $\mathrm{g}^{-1}$ para acerola, $9.397 \mathrm{~g} \mathrm{~g}^{-1} \mathrm{DPPH}$ e $7,8 \mu \mathrm{M}$ trolox g $\mathrm{g}^{-1}$ para cajá, $7.142 \mathrm{~g} \mathrm{~g}^{-1}$ DPPH e 11,2 $\mu \mathrm{M}$ trolox $\mathrm{g}^{-1}$ para caju e $478 \mathrm{~g} \mathrm{~g}^{-1} \mathrm{DPPH}$ e $153 \mu \mathrm{M}$ trolox g-1 para camu-camu, pelos métodos DPPH• e ABTS ${ }^{\bullet}$, respectivamente.

Pereira et al. (2014), no desenvolvimento de bebida mista à base de água de coco, polpa de abacaxi e acerola, encontraram a maior atividade antioxidante para a formulação contendo $65 \%$ de água de coco, $15 \%$ de polpa de abacaxi e $20 \%$ de polpa de acerola, correspondendo a 12,67 $\mu \mathrm{M}$ trolox $\mathrm{g}^{-1}$ de suco, pelo método ABTS ${ }^{\bullet}$. Observou-se que a formulação F1 apresentou valor superior, no tempo zero, quando comparado àquele encontrado por Pereira et al. (2014).

Dionisio et al. (2016), ao estudarem a estabilidade de uma bebida funcional pasteurizada à base de yacon e frutas tropicais (camu-camu, acerola, caju, cajá, açaí e abacaxi), observaram, para a atividade antioxidante total pelo método do ABTS ${ }^{\bullet+}$, valor de 10,46 $\mu \mathrm{M}$ trolox $\mathrm{g}^{-1}$ para o tempo zero e 8,70 $\mu \mathrm{M}$ trolox $\mathrm{g}^{-1}$ para 225 dias de armazenamento, indicando perdas para esta característica na bebida em armazenamento refrigerado.

Observou-se que, embora tenham sido determinados maiores teores de carotenoides totais e antocianinas totais para a formulação $F 1$, isso não resultou em maior capacidade antioxidante para essa formulação (Tabela 2). Esse fato pode ser explicado pela presença de outros constituintes, tais como ácido ascórbico, compostos fenólicos, açúcares e outros compostos redutores que interferem nos testes de atividade antioxidante (REZAIRE et al., 2014). Alguns desses constituintes podem, também, contribuir de maneira mais significativa para a capacidade antioxidante (ROJAS-BARQUERA; NAVÁEZ-CUENCA, 2009).

\section{Conclusões}

Os sucos tropicais mistos obtidos se mostraram adequados em termos da legislação vigente, no que se refere à acidez titulável e aos sólidos solúveis, ao longo de 365 dias de armazenamento congelado das polpas correspondentes. Com relação às características físicas e químicas avaliadas, as variações observadas nos sucos tropicais mistos foram consideradas irrelevantes.

Quanto à presença de constituintes bioativos, o suco tropical misto F2, por ter uma concentração maior de açaí e cajá, apresentou maiores teores de antocianinas totais e carotenoides totais. As antocianinas totais mostraram-se mais instáveis que os carotenoides totais durante armazenamento, com perdas significativas de $44,35 \%$ e $73,48 \%$, para as formulações F1 e F2, respectivamente. Ambas as formulações estudadas apresentaram significativa capacidade antioxidante durante 365 dias, com destaque para a formulação F1.

De maneira geral, observou-se que o armazenamento sob congelamento foi eficaz na manutenção das características químicas e das capacidades antioxidantes das polpas de frutas mistas estudadas, avaliadas a partir de sucos tropicais mistos formulados com água e açúcar.

\section{Referências}

BARBOSA, S. J. Qualidade de suco em pó de misturas de frutas obtido por spray drying. 2010. 107 f. Dissertação (Mestrado em Produção Vegetal no Semiárido)-Universidade Estadual de Montes Claros, Janaúba, 2010.

BEZERRA, C. V.; SILVA, L. H. M.; COSTA, R. D. S.; MATTIETTO, R. A.; RODRIGUES, A. M. C. Comportamento reológico de suco misto elaborado com frutas tropicais. Brazilian Journal of Food Technology, Campinas, v. 16, n. 2, p. 155-162, 2013. http:// dx.doi.org/10.1590/S1981-67232013005000020.

BRAND-WILIAMS, W.; CUVELIER, M. E.; BERSET, C. Use of a free radical method to evaluate antioxidant activity. Food Science and Technology-Lebensmittel-Wissenschaft \& Technologie, London, v. 28, n. 1, p. 25-30, 1995.

BRASIL. Instrução Normativa $n^{\circ} 1$, de 7 de janeiro de 2000. Regulamento técnico geral para fixação dos padrões de identidade e qualidade para polpa de fruta. Diário Oficial [da] República Federativa do Brasil, Brasília, DF, 10 jan. 2000. Seção 1, p. 54. Disponível em: <http://www. agricultura.gov.br>. Acesso em: 18 fev. 2016. 
Estudo da estabilidade de polpas de frutas tropicais mistas congeladas utilizadas na formulação de bebidas Carvalho, A. V. et al.

BRASIL. Instrução Normativa n 12, de 4 de setembro de 2003. Regulamento técnico para fixação dos padrões de identidade e qualidade gerais para suco tropical. Diário Oficial [da] República Federativa do Brasil, Brasília, DF, 09 set. 2003. Seção 1, p. 2. Disponível em: <http://www.agricultura.gov.br>. Acesso em: 18 fev. 2016

CARVALHO, A. V.; CAVALCANTE, M. A.; SANTANA, C. L.; ALVES, R. M. Características físicas, químicas e atividade antioxidante de frutos de matrizes de cajazeira no estado do Pará. Alimentos e Nutrição, Araraquara, v. 22, n. 1, p. 45-53, 2011.

CUNNIFF, P. (Ed.). Official methods of analysis of the Association of Official Analytical Chemists. 16th ed. Gaithersburg: AOAC, 1997.

DIONISIO, A. P.; WURLITZER, N. J.; GOES, T. S.; BORGES, M. F.; GARRUTI, D.; ARAUJO, I. M. S. Estabilidade de uma bebida funcional de frutas tropicais e yacon (Smallanthus sonchifolius) durante o armazenamento sob refrigeração. Archivos Latinoamericanos de Nutricion, Venezuela, v. 66, n. 2, p. 148-155, 2016

FREITAS, C. A. S.; MAIA, G. A.; COSTA, J. M. C.; FIGUEIREDO, R. W.; SOUSA, P. H. M.; FERNANDES, A. G. Estabilidade dos carotenoides, antocianinas e vitamina $C$ presentes no suco tropical de acerola (Malpighia emarginata DC.) adoçado envasado pelos processos hot-fill e asséptico. Ciência e Agrotecnologia, Lavras, v. 30, n. 5, p. 942-949, 2006.

GIUSTI, M. M.; WROLSTAD, R. E. Characterization and mesasurement of anthocyanins by UV-visible spectroscopy. In: WROLSTAD, R. E. (Ed.). Current protocols in food analytical chemistry. New York: Jonhn Willey \& Sons, 2001.

HAMANO, P. S.; MERCADANTE, A. Z. Compositions of carotenoids from commercial products of caja (Spondias lutea). Journal of Food Composition and Analysis, San Diego, v. 14, n. 4, p. 335-343, 2001. http://dx.doi.org/10.1006/jfca.2000.0975.

HERNÁNDEZ, M. S.; CARRILO, M.; BERRERA, J. Camu-camu (Myrciaria dubia Kunth McVaugh). In: YAHIA, E. M. (Ed.). Postharvest biology and technology of tropical and subtropical fruits. Cambridge: Woodhead Publishing, 2011. Chap. 16, p. 352-362.

LARRAURI, J. A.; RUPÉREZ, P.; SAURA-CALIXTO, F. Effect of drying temperature on the stabilitity of polyphenols and antioxidant activity of red grape pomace peels. Journal of Agriculture and Food Chemistry, Washington, v. 45, n. 4, p. 1390-1393, 1997. http://dx.doi.org/10.1021/jf960282f.

LOPES, T. J.; XAVIER, M. F.; QUADRI, M. G. N.; QUADRI, M. B. Antocianinas: uma breve revisão das características estruturais e da estabilidade. Revista Brasileira de Agrociência, Pelotas, v. 13m, n. 3, p. 291-297, 2007.

MAIA, G. A.; SOUSA, P. H. M.; LIMA, A. S. Processamento de sucos de frutas tropicais. Fortaleza: Edições UFC, 2007. 320 p.
MATTIETTO, R. A.; LOPES, A. S.; MENEZES, H. C. Estabilidade do néctar misto de cajá e umbu. Ciência e Tecnologia de Alimentos, Campinas, v. 27, n. 3, p. 456-463, 2007.

MILLER, N. J.; DIPLOCK, A. T.; RICE-EVANS, C.; DAVIES, M. J.; GOPINATHAN, V.; MILNER, A. A novel method for measuring antioxidant capacity and its application to monitoring the antioxidant status in premature neonates. Clinical Science, London, v. 84, p. 407-412, 1993.

NAKANO, L. A.; CANDÉA, I. V.; MATTIETTO, R. A.; GOMES, F. S.; MATTA, V. M. Compostos bioativos em polpas de frutas da Amazônia. In: CONGRESSO BRASILEIRO DE CIÊNCIA E TECNOLOGIA DE ALIMENTOS, 23., 2012, Campinas. Campinas: UNICAMP, 2012. Disponível em: <http://ainfo.cnptia.embrapa. br/digital/bitstream/item/79071/1/2012-128-NAKANO. pdf>.

Acesso em: 14 jan. 2016

NEVES, L. C.; BENEDETTE, R. M.; TOSIN, J. M.; CHAGAS, E. A.; SILVA, V. X.; PRILL, M. A. S.; ROBERTO, S. R. Produção de blends a partir de frutos tropicais e nativos da Amazônia. Revista Brasileira de Fruticultura, Jaboticabal, v. 33, n. 1, p. 187-197, 2011

ORDOÑEZ PEREDA, J. A. Tecnologia de alimentos: componentes dos alimentos e processos. Porto Alegre: Artmed, 2005. v. 1, 294 p.

PAZ, M.; GÚllon, P.; BARROSO, M. F.; CARVALHO, A. P.; DOMINGUES, V. F.; PEREIRA, A. C. S.; SIQUEIRA, A. M. A.; FARIAS, J. M.; MAIA, G. A.; FIGUEIREDO, R. W.; SOUZA, P. H. $M$. Desenvolvimento de bebida mista à base de água de coco, polpa de acabaxi e acerola. Archivos Latinoamericanos de Nutricion, Venezuela, v. 59, n. 4, p. 41-47, 2009.

PEREIRA, A. C. S.; DIONÍSIO, A. P.; WURLITZER, N. J.; ALVES, R. E.; BRITO, E. S.; SILVA, A. M. O.; BRASIL, I. M.; MANCINI FILHO, J. Effect of antioxidant potential of tropical fruit juices on antioxidant enzyme profiles and lipid peroxidation in rats. Food Chemistry, Barking, v. 157, p. 179-185, 2014.

REZAIRE, A.; ROBINSON, J. C.; BEREAU, D.; VERBAERE, A.; SOMMERER, N.; KHAN, M. K.; DURAND, P.; PROST, E.; FILSLYCAON, B. Amazonian palm Oenocarpus bataua ("patawa"): chemical andbiological antioxidant activity: phytochemical composition. Food Chemistry, Barking, v. 149, p. 62-70, 2014.

RODRIGUEZ-AMAYA, D. B. A guide to carotenoid analysis in foods. Washington: ILSI Press, 1999. 64 p.

ROJAS-BARQUERA, D.; NARVÁEZ-CUENCA, C. E. Determinación de vitamina $C$, compuestos fenólicos totales y actividad antioxidante de frutas de guayaba culivadas en Colombia. Química Nova, São Paulo, v. 32, n. 9, p. 2336-2340, 2009.

RUFINO, M. S. M.; ALVES, R. E.; BRITO, E. S.; MORAIS, S. M.; SAMPAIO, C. G.; PÉREZ-JIMÉNEZ, J.; SAURA-CALIXTO, F. D. Metodologia científica: determinação da atividade antioxidante total em frutas pela captura do radical livre ABTS. Fortaleza: Embrapa Agroindústria Tropical, 2007. (Comunicado Técnico, 128). 
Estudo da estabilidade de polpas de frutas tropicais mistas congeladas utilizadas na formulação de bebidas

Carvalho, A. V. et al.

RUFINO, M. S. M.; ALVES, R. E.; BRITO, E. S.; PÉREZ-JIMÉNEZ, J.; SAURA-CALIXTO, F.; MANCINI-FILHO, J. Bioactive compounds and antioxidant capacities of 18 non-traditional tropical fruits from Brazil. Food Chemistry, London, v. 121, n. 4, p. 996-1002, 2010. http://dx.doi.org/10.1016/j.foodchem.2010.01.037.

SAS INSTITUTE. SAS for Windows: versão 8.0 SAS ${ }^{\circledR}$. SAS user guide. Carry: Statistical Analysis System Institute, 1999.
VEGARA, S.; MENA, P.; MARTI, N.; SAURA, D.; VALERO, M. Approaches to understanding the contribution of anthocyanins to the antioxidant capacity of pasteurized pomegranate juices. Food Chemistry, London, v. 141, n. 3, p. 1630-1636, 2013. PMid:23870870. http://dx.doi.org/10.1016/j.foodchem.2013.05.015.

WILLIANS, S. (Ed.). Official methods of analysis of the Association of Official Analytical Chemists. 14th ed. Arlington: AOAC, 1984. 